\title{
Surface optical phonons in GaAs nanowires grown by Ga-assisted chemical beam epitaxy
}

\author{
C. García Núñez, ${ }^{1, a)}$ A. F. Braña, ${ }^{1}$ J. L. Pau, ${ }^{1}$ D. Ghita, ${ }^{1}$ B. J. García, ${ }^{1}$ G. Shen, ${ }^{2}$ \\ D. S. Wilbert, ${ }^{2}$ S. M. Kim, ${ }^{2}$ and P. Kung ${ }^{2}$ \\ ${ }^{1}$ Grupo de Electrónica y Semiconductores, Departamento de Física Aplicada, \\ Universidad Autónoma de Madrid, 28049 Madrid, Spain \\ ${ }^{2}$ Department of Electrical and Computer Engineering, The University of Alabama, Tuscaloosa, \\ Alabama 35487, USA
}

(Received 10 December 2013; accepted 27 December 2013; published online 21 January 2014)

\begin{abstract}
Surface optical (SO) phonons were studied by Raman spectroscopy in GaAs nanowires (NWs) grown by Ga-assisted chemical beam epitaxy on oxidized Si(111) substrates. NW diameters and lengths ranging between 40 and $65 \mathrm{~nm}$ and between 0.3 and $1.3 \mu \mathrm{m}$, respectively, were observed under different growth conditions. The analysis of the Raman peak shape associated to either longitudinal or surface optical modes gave important information about the crystal quality of grown NWs. Phonon confinement model was used to calculate the density of defects as a function of the NW diameter resulting in values between 0.02 and 0.03 defects $/ \mathrm{nm}$, indicating the high uniformity obtained on NWs cross section size during growth. SO mode shows frequency downshifting as NW diameter decreases, this shift being sensitive to NW sidewall oxidation. The wavevector necessary to activate SO phonon was used to estimate the NW facet roughness responsible for SO shift. (C) 2014 AIP Publishing LLC. [http://dx.doi.org/10.1063/1.4862742]
\end{abstract}

\section{INTRODUCTION}

Nanowires (NWs) are considered as interesting crystalline nanostructures to study low dimensional physics and other properties arising from their high surface-to-volume ratio or carrier quantum confinement. These intrinsic properties give NWs high sensitivity to surface effects, allowing electrical response rates which are attractive characteristics to improve efficiency of common devices used in optoelectronics, electronics, or sensing. ${ }^{1-4}$

GaAs NWs have been synthesized using different techniques such as molecular beam epitaxy (MBE), ${ }^{5}$ metal organic chemical vapor deposition (MOCVD), ${ }^{6}$ and chemical beam epitaxy (CBE). ${ }^{7}$ Growth mechanism of GaAs NWs is known as vapor-liquid-solid (VLS) method, which was first proposed by Wagner and Ellis ${ }^{8}$ utilizing pre-deposited $\mathrm{Au}$ droplets as liquid catalyst to assist the Si NWs growth along a preferential direction fixed by the substrate. Au has been widely used as a catalyst for the growth of various NWs, ${ }^{9-11}$ although it was demonstrated its use can hinder electrical properties of NWs by generating traps in the energy gap of the semiconductor material. ${ }^{12,13} \mathrm{Ga}$ catalyst was proposed as a potential alternative, allowing the growth of Ga-assisted GaAs NWs by VLS, also called self-catalyst growth. ${ }^{14}$ When the Ga liquid droplet becomes supersaturated of As coming from the gas ambient, GaAs solid phase starts growing underneath the droplet, forming a NW whose diameter is limited by the droplet size. $\operatorname{Si}(111)$ is an ideal substrate to grow vertically aligned GaAs NWs ${ }^{15,16}$ because of:

a) The native oxide on the substrate surface presents pinholes where Ga droplets can be preferentially bonded.

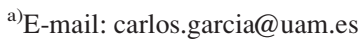

b) Silicon substrates could allow for silicon and GaAs device integration.

c) The high aspect ratio of the NWs and the low GaAs lattice mismatch (4\%) allow the coherent growth of GaAs NWs with critical diameters up to $110 \mathrm{~nm} .^{17}$

However, one major problem found in VLS grown NWs is the presence of structural defects - such as twins, voids, or stacking faults - generated around the interface between the NW and the catalyst during the growth.

Raman spectroscopy, as a non-destructive contactless characterization technique, allows us to analyze surface properties (surface phonon existence), structural quality (NW strain and density of structural defects), thermal and quantum confinement effects, as a function of the NW dimensions. ${ }^{18-21}$ Tensile and compressive forces can be identified in Raman spectra as red and blue energy shifts, respectively. The existence of defects in the NW lattice structure leads to a relaxation of the Raman selection rules at the Brillouin zone (BZ) centre ( $\Gamma$ point, wavevector $q=0$ ) by a quantity $(\delta q)$ proportional to the inverse of the NW diameter $\left(\delta q \sim \pi / d_{\mathrm{NW}}\right){ }^{22}$ this breaking of the selection rules allows not only the observation of forbidden modes in different backscattering configurations but also peak shifts and asymmetrical broadening of the Raman line shape. For III-V compound semiconductors with zincblende (ZB) structure, transverse (TO) and longitudinal (LO) optical modes are split at $q \sim 0$ due to the polar nature of the crystal (long range Coulomb forces). TO and LO vibrational modes have been observed in GaAs NWs under specific backscattering geometries, ${ }^{23}$ being strongly affected by lattice defects.

As mentioned before, Raman peak shifts can also be influenced by tensile and compressive strains generated along the NW especially in polytypism structures formed by both phases $\mathrm{ZB}$ and wurtzite (WZ), where strain can be 
characterized by a peak shift towards lower and higher Raman wavenumbers, respectively. ${ }^{23}$ Quantum confinement could also lead to a peak shift toward lower energies; as demonstrated by Campbell and Fauchet calculations, NWs with diameters below $3 \mathrm{~nm}$ are necessary to show a significant peak shift up to $4 \mathrm{~cm}^{-1} \cdot{ }^{24}$ Furthermore, the excitation laser power can induce shifts on the TO and LO peaks towards lower energies, due to sample heating. ${ }^{19,25}$

A third Raman peak could be observed at Raman frequencies located between TO and LO peaks, which is usually attributed to a surface optical (SO) phonon. This vibrational mode was first proposed by Fuchs and Kilemer ${ }^{26}$ who considered the SO mode as a macroscopic optical phonon whose propagation and penetration depth were connected with a macroscopic electric field. SO phonons can be generated at the interface between different materials, propagating along their interfaces with a wavevector $q$. Atom vibrations involved in the propagation of the SO have amplitudes which are confined to the near-surface region of the material. SO modes are generated by the breakdown of the translational symmetry of the surface potential, which in a NW is due to the surface roughness or to different diameters along the NW length. Therefore, this phonon can be actively associated with surface modulation (roughness), cross section shape (hexagonal, cylindrical, or square), and backscattering geometry. There are a few reports on SO phonons observed in NWs composed of various semiconductor materials such as InGaN/GaN multi-quantumwell nanopillars, ${ }^{27} \mathrm{AlN},{ }^{28} \mathrm{GaN}^{29} \mathrm{InN}^{30} \mathrm{GaP},{ }^{31}$ or GaAs. ${ }^{16,18}$ Some of above works show relevant information about the NW structure and surface quality of the NWs extracted from the Raman analysis of the SO peak.

In this work, we present a thorough study of the Raman line-shape and peak shift of TO, SO, and LO phonon modes measured by Raman spectroscopy in GaAs NWs with different diameters. NWs were grown by CBE under specific conditions which allow us to control NW diameter, length, and crystal phase. ${ }^{16}$ A theoretical model was used to simulate LO peak line shape, in order to extract the density of defects in the lattice structure of a single GaAs NW through the estimation of the correlation length $(L)$, defined as the distance between two adjacent lattice defects such as vacancies, interstitials, and dislocations.

In addition, we investigate the mechanism responsible for the SO phonon generation by the Raman study of the length scale parameter $(\lambda)$ or also called SO wavelength perturbation. ${ }^{31}$ This parameter is defined as the distance between two adjacent morphological defects found along the surface of the NW, its value being extracted from the difference between the SO and LO peak frequencies, and later corroborated by transmission electron microscopy (TEM) images. As NW sidewall oxidation could also produce SO frequency shifts, caused by the propagation of the SO mode through the interface formed by oxide and the NW, the influence of a native oxide layer has been considered.

\section{EXPERIMENTAL}

Vertically aligned GaAs NWs were grown on oxidized $\mathrm{Si}(111)$ substrates by means of Ga-assisted CBE using triethylgallium (TEGa) and tertiarybutylarsine (TBAs) as precursors for Ga and As atoms, respectively. TBAs flux was directed to the substrate using a high temperature gas cell $\left(T=820^{\circ} \mathrm{C}\right)$, while uncracked TEGa was introduced through a low temperature gas cell $\left(T=80^{\circ} \mathrm{C}\right)$. Si(111) substrates were etched in a $10 \% \mathrm{HF}$-ethanol solution, followed by rinsing in deionised water and dried with $\mathrm{N}_{2}$. The short exposure to ambient air before loading the sample into the ultra-high vacuum system leads to the formation of a thin rough native oxide layer with a thickness of about $0.8 \mathrm{~nm}$, as measured by spectroscopic ellipsometry. After sample loading, the substrate was heated up to $T_{\mathrm{S}}=650^{\circ} \mathrm{C}$ during $5 \mathrm{~min}$ to allow for impurity desorption. $T_{\mathrm{S}}$ was then reduced to $580{ }^{\circ} \mathrm{C}$ to start a pre-deposition of $\mathrm{Ga}$ atoms for $15 \mathrm{~s}$ (Ga coverage about 7 monolayers) followed by a stabilization step (90 s) to allow for Ga droplet formation and migration to pinholes in the oxide. For NW growth, the III/V ratio was set slightly larger than unity, with an equivalent two-dimensional growth rate $v_{\mathrm{g}}=0.5 \mu \mathrm{m} / \mathrm{h}$, as determined by reflection high-energy electron diffraction (RHEED) frequency measurements on $\mathrm{GaAs}(001)$ substrates.

Compositional and structural properties of individual GaAs NWs were analyzed by means of energy dispersive X-ray spectroscopy (EDS), TEM, and Raman spectroscopy. For EDS and TEM measurements, NWs samples $\left(1 \times 1 \mathrm{~cm}^{2}\right)$ were dipped in ethanol and sonicated in order to remove NWs from the Si substrate. After that, a micro-drop of the obtained solution was deposited on a holey carbon copper grid.

Optical phonon modes in GaAs NWs structures were analyzed by means of confocal Raman spectroscopy at roomtemperature, using the $532 \mathrm{~nm}$ wavelength of a diode-pumped solid-state frequency doubled $\mathrm{Nd}: \mathrm{YVO}_{4}$ laser focused on the sample surface through a $100 \times$ objective operating at different low excitation powers, between $12 \mu \mathrm{W}$ and $5.6 \mathrm{~mW}$, to avoid any noticeable NW heating.

For Raman characterization, GaAs NWs growth direction was assumed to be $[-1-1-1],{ }^{16}$ then the NW coordinate system is set with the $\mathrm{z}$ axis along the growth direction, and the [-211] and [0-11] directions corresponding to the NW edge and facet perpendicular directions, respectively, being contained in the xy plane. Therefore, Raman configuration was $\mathrm{z}(\mathrm{xy}, \mathrm{xy}) \mathrm{z}$ which means the incident beam was focused onto the (111) surface (NW front), while no special care was taken to align the polarization vector relative to the NW facets. As the laser spot was focused using a $100 \times$ objective with a spot of a few microns in size, around 3 or 4 NWs were irradiated during each measurement; this configuration has been demonstrated to allow both TO and LO phonon modes. ${ }^{23}$

\section{RESULTS AND DISCUSSION}

Several samples were grown for $T_{\mathrm{S}}$ values of 580,600 , and $620^{\circ} \mathrm{C}$, using growth times of 450 and $900 \mathrm{~s}$. All the samples showed randomly distributed NWs, mainly aligned along the perpendicular direction to the substrate surface, showing a hexagonal footprint with an average NW diameter between 40 and $65 \mathrm{~nm}$, and terminated by a metallic $\mathrm{Ga}$ droplet. Figure 1 shows a typical scanning electron 


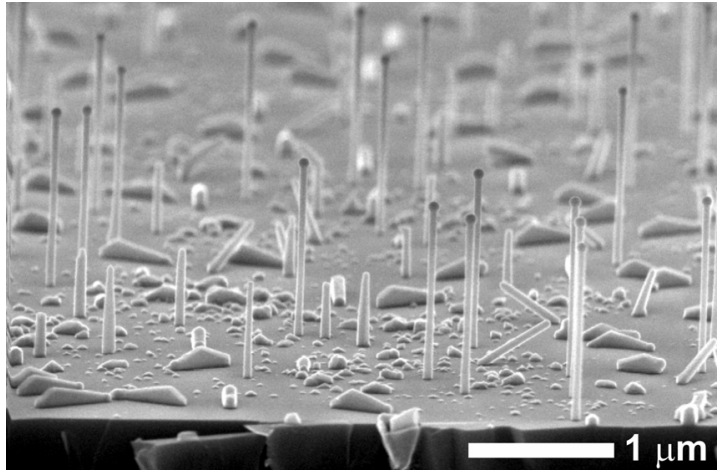

FIG. 1. SEM image of a sample grown at $580^{\circ} \mathrm{C}$ for $900 \mathrm{~s}$, tilted $80^{\circ}$ away from the normal to the substrate surface.

microscopy (SEM) image corresponding to a sample grown at $T_{\mathrm{S}}=580^{\circ} \mathrm{C}$ during $900 \mathrm{~s}$, obtained using a tilt angle of $80^{\circ}$ away from the sample normal.

Samples grown at the higher temperatures present lower density of NWs, but with larger diameters $\left(d_{\mathrm{NW}} \sim 65 \mathrm{~nm}\right.$ at $\left.T_{\mathrm{s}}=620^{\circ} \mathrm{C}\right) .{ }^{16}$ This trend can be explained because the mobility of $\mathrm{Ga}$ atoms over the $\mathrm{Si}(111)$ surface increases with $T_{\mathrm{S}}$, enhancing the trapping efficiency of $\mathrm{Ga}$ atoms at $\mathrm{Ga}$ droplets and thus enlarging the NW influence area at higher temperatures.

Figure 2(a) shows the EDS linear scan taken in scanning TEM mode (STEM) along a $100 \mathrm{~nm}$ path from the final droplet to the body of the NW (grown at $T_{\mathrm{S}}=600^{\circ} \mathrm{C}$ for $900 \mathrm{~s}$ ), as shown in Figure 2(b) superimposed to a TEM + STEM collage image. The used system allows to carry out chemical characterization with a lateral resolution of around $2-3 \mathrm{~nm}$. EDS chemical analysis revealed the droplet was essentially composed of $\mathrm{Ga}$, while the NW body was stoichiometric GaAs. In addition, a nearly constant signal related to $\mathrm{O}$ is seen along the whole scan, in both the NW and the droplet regions, which could be related to different factors such as $\mathrm{SiO}_{2}$ reduction during the $\mathrm{Ga}$ droplet formation or native oxide layer grown during sample storage due to air exposure.

Regardless of substrate temperature and growth time, high resolution TEM (HRTEM) imaging (Figure 2(c)) and its fast Fourier transform (FFT) (Figure 2(d)) demonstrated that NWs were grown along the $\langle 111\rangle$ direction with a ZB structure, clearly identifying the $\{111\},\{002\}$, and $\{11-1\}$ families of planes, associated to such structure. A detailed TEM study focused on the growth details of pure ZB GaAs NWs has been reported elsewhere. ${ }^{16}$

Figure 3 shows three Raman spectra corresponding to samples grown at $T_{\mathrm{S}}=580^{\circ} \mathrm{C}$ during $900 \mathrm{~s}\left(d_{\mathrm{NW}}=40 \mathrm{~nm}\right)$, $T_{\mathrm{S}}=600^{\circ} \mathrm{C}$ during $450 \mathrm{~s}\left(d_{\mathrm{NW}}=54 \mathrm{~nm}\right)$ and $T_{\mathrm{S}}=600^{\circ} \mathrm{C}$ for $900 \mathrm{~s}\left(d_{\mathrm{NW}}=57 \mathrm{~nm}\right)$. TO and LO characteristic peaks of ZB GaAs are clearly observed at $268 \pm 1$ and $288 \pm 1 \mathrm{~cm}^{-1}$, respectively. The measured $\mathrm{TO}$ and $\mathrm{LO}$ frequency values are slightly shifted to lower values respect to the usual bulk ZB $\operatorname{GaAs}(111)$ ones $\left(\mathrm{TO}=269 \mathrm{~cm}^{-1}\right.$ and $\left.\mathrm{LO}=292 \mathrm{~cm}^{-1}\right)$. The small observed shift could be related to the strain induced by the lattice mismatch, because TO and LO peak shifts were found to be almost independent on the laser excitation power-within the range of laser excitation power used in (a)
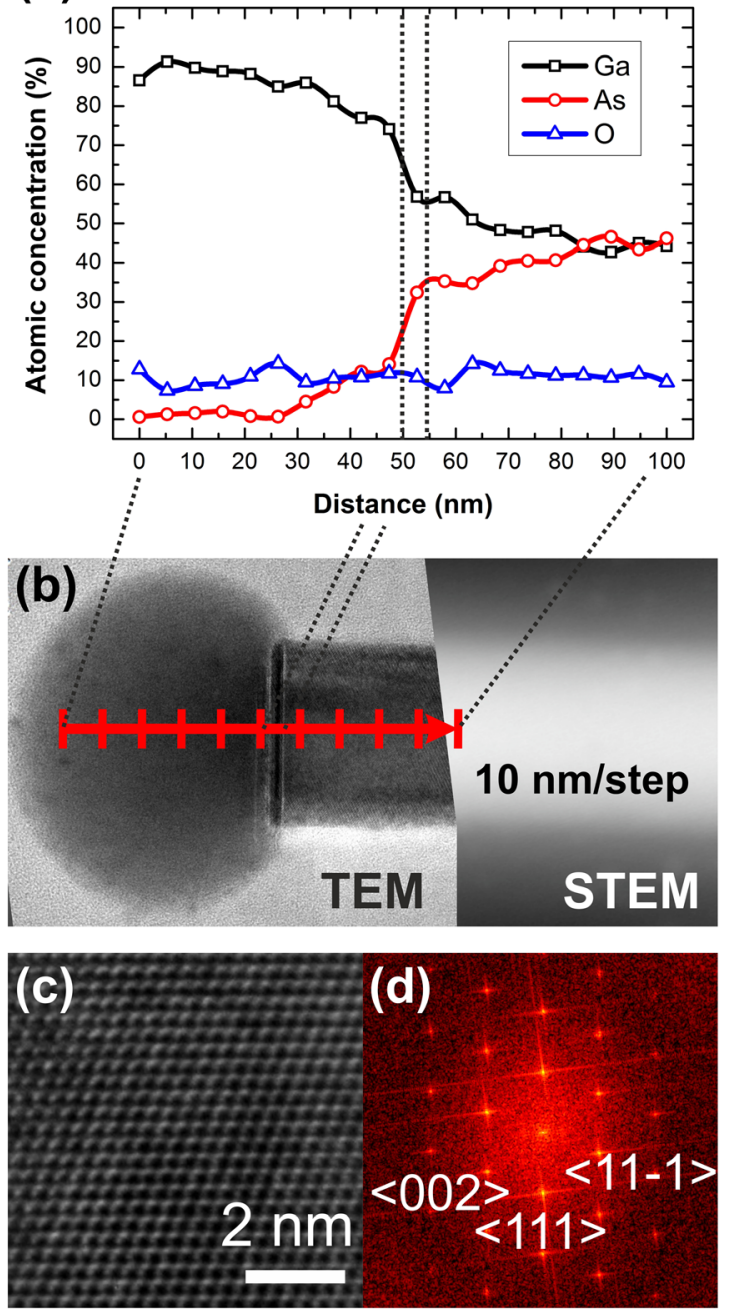

FIG. 2. Single GaAs NW grown at $600^{\circ} \mathrm{C}$ : (a) EDS spectra showing Ga, As, and $\mathrm{O}$ contents along a linear scan from the Ga droplet to the NW. (b) TEM image overview: TEM and STEM collage, (c) HRTEM image, and (d) HRTEM 2D FFT image.

our Raman experiments-therefore, no heating effects were noticed (Figure 4). ${ }^{19,21}$

TO and LO peaks shown in Figure 3 exhibit small full width at half-maximum (FWHM) values, around $5 \mathrm{~cm}^{-1}$, indicating NWs have a high crystal quality. In addition, the ratio between the intensities of both peaks $\left(I_{\mathrm{TO}} / I_{\mathrm{LO}}\right)$ is found to be close to 1.5 , higher values being usually related to $\mathrm{WZ}$ GaAs NWs $(>1.5)$, while much lower values are found in the case of GaAs epilayers $(<0.1)$, this ratio depending on the crystal orientation, measurement geometry, and surface electric field. ${ }^{25}$

Raman spectra of GaAs NWs confirm the existence of pure ZB under the backscattering geometry described in Sec. II. The lack of peaks associated to the wurzite structure such as $E_{2}{ }^{\mathrm{H}}$, which is expected to be observed at $259 \mathrm{~cm}^{-1},{ }^{16,23}$ is in good agreement with the absence of WZ phase in analyzed TEM images.

The third peak observed between TO and LO peaks is attributed to a SO phonon, due to the following characteristics associated to this vibrational mode. SO modes exhibit dispersion, which means the SO frequency $\left(\omega_{\mathrm{SO}}\right)$ is a function of 


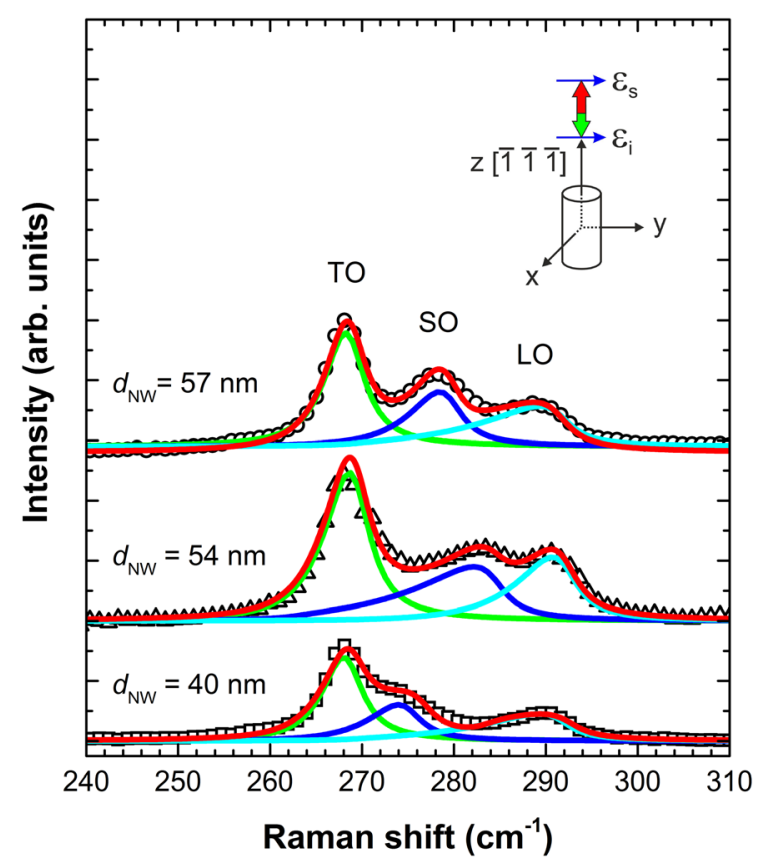

FIG. 3. Raman spectra of GaAs NWs with different diameters. Inset: schematic of the bakscattering configuration $\mathrm{z}(\mathrm{xy}, \mathrm{xy})-\mathrm{z} . \varepsilon_{\mathrm{i}}$ and $\varepsilon_{\mathrm{s}}$ are the electric field directions of the incident and scattered beams.

the wavevector $q$. First condition a SO phonon has to satisfy is that for any $q$, the $\omega_{\mathrm{SO}}$ value must lie between the $\omega_{\mathrm{TO}}$ and $\omega_{\mathrm{LO}}$ ones. On the other hand, $\omega_{\mathrm{SO}}$ should also be dependent on both $d_{\mathrm{NW}}$ and the relative permittivity of the surrounding environment $\left(\varepsilon_{\mathrm{m}}\right)$. Therefore, the analysis of SO peak will allow us to extract information about surface effects on the vibration mode as well as detecting changes along the NW surface morphology. A further discussion about the $\omega_{\text {SO }}$ characteristics will be shown below.

Lattice disorder in the NW structure and also lowdimensional effects can be studied by means of the Raman line-shape analysis using the phonon confinement model (PCM), also known as "special correlation model." This model, first proposed by Richter to account for the observed

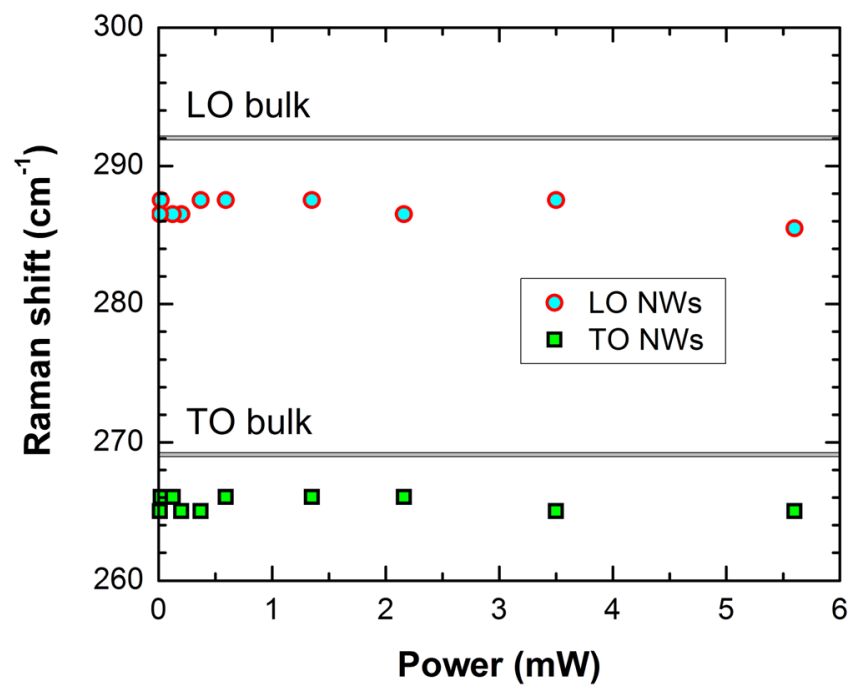

FIG. 4. Raman shifts for the LO and TO modes in GaAs NWs grown at $580^{\circ} \mathrm{C}$. Shifts corresponding to the $\mathrm{GaAs}(111)$ epitaxial bulk layer values are also shown. shift and broadening in microcrystalline silicon, ${ }^{32}$ and further developed by Campbell ${ }^{24}$ to be applied to cylindrically shaped NWs and thin films, has been used to analyze Raman line shapes in NWs composed by different semiconductor materials such as $\mathrm{GaP},{ }^{31} \mathrm{Si}^{20}$ InAs, or GaAs. ${ }^{18}$ This model takes into account the contributions of phonons away from the first Brillouin zone, which are responsible for the modification of the Raman spectrum line shape. PCM has also been used to study LO modes showing broadening and asymmetric peak-shapes, because the existence of a SO mode close to the LO peak can distort its shape. However, PCM has rarely been used for the TO mode; due to its low dispersion, this peak presents symmetric Lorentzian shape and therefore neither structural disorder nor quantum confinement effects can be studied for this vibrational mode. According to PCM, considering a set of NWs with a given distribution of diameters, but constant along their length, Raman intensity can be expressed as

$$
I(\omega) \cong \int_{\mathrm{BZ}} \frac{\left|C\left(q_{0}, q\right)\right|^{2}}{\left[\omega-\omega_{0}(q)\right]^{2}+\left[\Gamma_{0} / 2\right]^{2}} d q^{3},
$$

where $\omega$ is the incident photon frequency, $d q^{3} \sim q d q$ for NWs, ${ }^{19}\left|C\left(q_{0}, q\right)\right|^{2}$ is the Fourier coefficient of a confinement function which could depend on the structure shape and it is a function of the wavevector $q$ centered at $q_{0},{ }^{25} 1 / \Gamma_{0}$ is the phonon lifetime $\left(\Gamma_{0}\right.$ represents the natural FWHM of the bulk GaAs phonon and takes a value about $4.5 \mathrm{~cm}^{-1}$ ), while $\omega_{0}(q)$ is the phonon dispersion relationship. Although both $\Gamma_{0}$ and $\omega_{0}(q)$ are functions of the NW temperature, the use of a low laser excitation power allows to discard any temperature dependence.

While Equation (1) was presented by Richter to analyze LO phonons along the $\langle 001\rangle$ directions, corresponding to the directions connecting the $\Gamma$ and $\mathrm{X}$ points in the first $\mathrm{BZ},{ }^{32}$ this expression will be also used in this work to accurately fit the line shapes of all three Raman modes (TO, SO, and LO) measured in GaAs NWs samples.

For a primitive cell including two atoms (such as GaAs) bonded by a force $C_{1}$ (see Figure 5), $\omega_{0}(q)$ can be expressed as $^{33}$

$$
\omega_{0}(q)=C_{1} \sqrt{\frac{M_{1}+M_{2}+\sqrt{M_{1}^{2}+M_{2}^{2}+2 M_{1} M_{2} \cos (q a)}}{M_{1} M_{2}}},
$$

where $M_{1}$ and $M_{2}$ are the averaged Ga (69.723) and As (74.922) atomic masses, $a$ is the spacing between adjacent planes with the same kind of atoms, and $q$ is the component of the wavevector along the direction perpendicular to these planes. Equation (2) can be deduced for waves propagating along a high-symmetry direction for which a single vibrating plane contains only a single type of atom; such directions are $\langle 111\rangle$ in $\mathrm{NaCl}$ and $\langle 100\rangle$ in $\mathrm{CsCl}$ crystals. ${ }^{34}$ Figures 5(a) and 5(b) show a schematic picture for the crystal structure of the cross section and lateral walls of a hexagonal ZB GaAs NW oriented along the [111]B direction. Figure 5(c) represents a 
phonon wave propagating along one of the $\langle 001\rangle$ directions, the $\{010\}$ family of planes containing only one type of atom (either $\mathrm{Ga}$ or As) with a spacing $a=a_{0} / 2=0.326 \mathrm{~nm}, a_{0}$ being the GaAs lattice parameter; both longitudinal and transverse phonons are represented, depending on the atomic vibration direction respect to the wavevector.

In the case of surface phonons, the SO mode is similar to those observed in bulk layers but with only atoms at the surface contributing to phonon propagation (Figure 5(d)).

When $q_{0}=0$, Fourier coefficient used in Equation (1) can be written as

$$
\begin{gathered}
|C(0, q)|^{2}=C_{0} \exp \left[-\frac{1}{2}(q A)^{2}\right], \\
A=L / 4 \pi \\
A=d_{\mathrm{NW}} / \alpha a_{0},
\end{gathered}
$$

where $C_{0}$ is a fitting parameter. The parameter $A$ has been defined by Campbell and $\mathrm{Adu}^{34}$ in different ways, as expressed in Eqs. (3b) and (3c), respectively. Equation (3b) uses the fitting parameter $L$, known as the correlation length (related to the distance between defects and equivalent to the grain size in polycrystals), while Eq. (3c) uses both parameters $d_{\mathrm{NW}}$ and $\alpha$, which are the NW diameter and the confinement coefficient, respectively. $L$ gives information about the size of the confinement region formed between two adjacent defects in the NW structure, whereas $\alpha$ sets the confinement scale in the NW due to its diameter size and is characteristic for each material and independent on the NW diameter. ${ }^{28}$

a) (111)B NW cross section

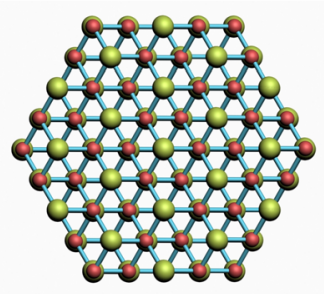

c) $\{010\}$ planes

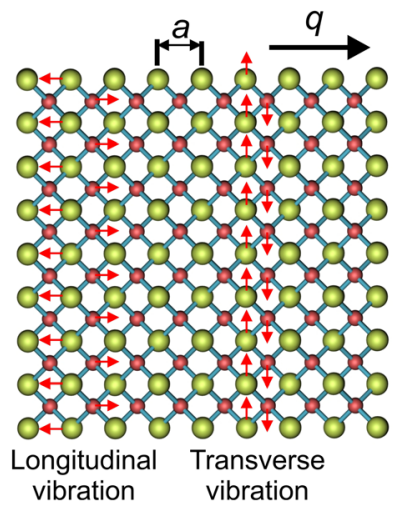

b) $\{110\} N W$ facet

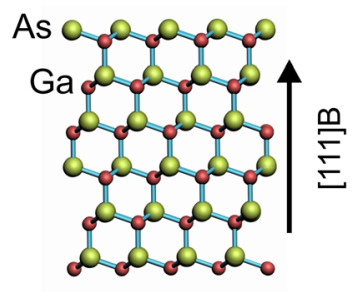

d) Surface vibration

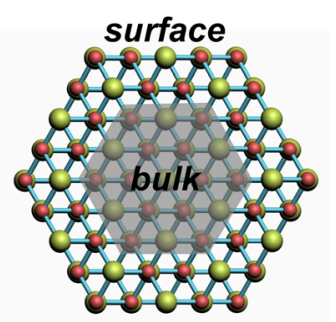

FIG. 5. Schematic model of a ZB GaAs NW. (a) NW cross section perpendicular to the Si $\langle 111\rangle$ growth direction; (b) one of the six NW facets belonging to the $\{110\}$ family of planes; (c) longitudinal and transverse vibrational modes; (d) only atoms in the surface structure are involved in the propagation of surface phonons.
The asymmetry, broadening, and downshifting of Raman peaks are mainly simulated by the Fourier coefficient (Eq. (3a)) using either $L$ or $d_{\mathrm{NW}}$ as fitting parameters, while the Raman intensity and peak position are determined by $C_{1}$ and $C_{0}$, respectively.

The Raman spectra shown in Figure 3 were simulated by the superposition of TO, LO, and SO phonon modes, each one fitted by using Eq. (1). The shape of the TO peak is almost a Lorentzian function, whereas in the case of SO and LO, they present some asymmetry and broadening. Because every peak has its own integral given by Eq. (1), every peak has its own set of fitting parameters given by $C_{0}, C_{1}, L$, and $\alpha$, where $L$ and $\alpha$ are obtained from Eqs. (3b) and (3c), respectively.

The analysis of the LO shape allows us to extract information about the disorder in the NW structure, according to PCM. A similar study was carried out by Begum et al. ${ }^{25}$ by means of a thorough analysis about the Raman line-shape in GaAs and InAs NWs grown by Au-assisted solid source MBE, estimating the density of defects in a single NW by using $L$. However, the density of defects in this kind of Auassisted growth resulted to be too high. Considering that $\alpha$ has been studied in several semiconductor NWs, but not yet in GaAs, the estimation of both parameters $\alpha$ and $L$ for GaAs NWs is promising in order to estimate the crystalline quality of NWs grown by different techniques and conditions.

LO peaks were fitted following the above procedure and using experimental values extracted from SEM images for $d_{\mathrm{NW}}$, obtaining parameters for $L$ and $\alpha$ which are shown in Table I. The correlation factor was obtained to be $r^{2} \geq 0.997$ for all fits, indicating that the model can accurately reproduce the LO peak shape.

The confinement coefficient value, $\alpha$, corresponding to GaAs NWs studied in this work was observed to be independent on $d_{\mathrm{NW}}$, taking values around $\alpha=23 \mathrm{~nm}^{-1}$, the value of this parameter being characteristic for each material. While it was previously reported to be $\alpha=6.3 \mathrm{~nm}^{-1}$ for $\mathrm{Si}$ $\mathrm{NWs},{ }^{21}$ to the best of our knowledge, its value has not been already estimated for GaAs NWs. Therefore, considering $\alpha$ is constant in Eq. (3c), the only factor responsible for the variation of $A$,- that is for changes in the asymmetry and broadening of the Raman peak-is $d_{\mathrm{NW}}$, which has been independently measured from SEM images.

On the other hand, the study of the correlation length, $L$, shows that $L$ increases with $d_{\mathrm{NW}}$. The density of defects can be estimated as $1 / L$. As shown in Table I, as $d_{\mathrm{NW}}$ increases (from 40 to $57 \mathrm{~nm}$ ) the defect concentration decreases (from 0.033 to $0.022 \mathrm{~nm}^{-1}$ ).

TABLE I. Parameters obtained after fitting LO shape ( $\alpha$ and $L$ ) using Eqs. (1)-(3) for GaAs NWs grown on $\mathrm{Si}(111)$ substrates with different diameters $\left(d_{\mathrm{NW}}\right)$.

\begin{tabular}{cccc}
\hline \hline $\begin{array}{c}d_{\mathrm{NW}} \pm 1 \\
(\mathrm{~nm})\end{array}$ & $\begin{array}{c}\alpha \pm 2 \\
\left(\mathrm{~nm}^{-1}\right)\end{array}$ & $\begin{array}{c}L \\
(\mathrm{~nm})\end{array}$ & $\begin{array}{c}\text { Defect density, } 1 / L \\
\left(\mathrm{~nm}^{-1}\right)\end{array}$ \\
\hline 40 & 23 & $30 \pm 3$ & $0.033 \pm 0.003$ \\
54 & 23 & $40 \pm 4$ & $0.025 \pm 0.003$ \\
57 & 23 & $45 \pm 5$ & $0.022 \pm 0.002$ \\
\hline \hline
\end{tabular}


Finally, the relationship obtained by combining Eqs. (3b) and (3c): $L=4 \pi d_{\mathrm{NW}} / \alpha a_{0}$, allows to calculate $L$ from simulated $\alpha$ values and vice versa; so obtained calculations differ between 5 and $10 \%$ from values shown in Table I, which is a good indicative that both Fourier coefficients were properly simulated.

SO peak line-shape and position study will be presented below. The $-\mathrm{z}(\mathrm{xy}, \mathrm{xy}) \mathrm{z}$ backscattering configuration offers one advantage over other possible configurations, such as, for example, $\mathrm{x}(\mathrm{y}, \mathrm{y})-\mathrm{x}$ where one of the $\{110\} \mathrm{NW}$ facets is illuminated, consisting on the empirical observation of a better defined SO band because of the large frequency shift between the LO and SO peaks.

Comparing LO and SO peak positions in Figure 3, the relative shift observed in the case of LO peak is below the resolution limit of $1 \mathrm{~cm}^{-1}$, while the SO peak position is more sensitive to either the NW diameter or the correlation length, showing a relative shift up to $9 \mathrm{~cm}^{-1}$.

From Figure 3, the SO peak shift dependence on NW diameter is not clear. Previously reported works ${ }^{18}$ observed an increasing downshift of $\omega_{\mathrm{SO}}$ with respect to the $\omega_{\mathrm{LO}}$ peak position as the NW diameter decreases, in such a way that when the SO extremely downshifts with respect to the LO peak, the latter becomes broad and asymmetric to compensate the lack of SO contribution.

It should be noted that because all NWs suffered a large exposure to ambient air before they were characterized, their surface could become oxidized. Surface oxidation process can induce changes in the SO frequency position due to the change of the relative permittivity at the GaAs NW surface (GaAs/oxide instead of GaAs/air). In order to better understand this effect, the frequency $\omega_{\text {SO }}$ can be calculated following a simple model which considers NWs as perfect cylinders surrounded by different environments; ${ }^{31}$ in this model, the SO frequency is given by expressions

$$
\begin{gathered}
\omega_{\mathrm{SO}}^{2}=\omega_{\mathrm{TO}}^{2}+\frac{\tilde{\omega}_{\mathrm{p}}^{2}}{\varepsilon_{\infty}+\varepsilon_{m} f\left(q r_{\mathrm{NW}}\right)}, \\
f\left(q r_{\mathrm{NW}}\right)=\frac{\left[I_{0}\left(q r_{\mathrm{NW}}\right) K_{1}\left(q r_{\mathrm{NW}}\right)\right]}{\left[I_{1}\left(q r_{\mathrm{NW}}\right) K_{0}\left(q r_{\mathrm{NW}}\right)\right]}, \\
\tilde{\omega}_{\mathrm{p}}^{2}=\left(\tilde{\omega}_{\mathrm{LO}}^{2}-\tilde{\omega}_{\mathrm{TO}}^{2}\right) \varepsilon_{\infty},
\end{gathered}
$$

where $\omega_{\mathrm{SO}}, \omega_{\mathrm{TO}}$, and $\omega_{\mathrm{LO}}$ are again the $\mathrm{SO}, \mathrm{TO}$, and $\mathrm{LO}$ phonon frequencies, respectively, $\tilde{\omega}_{\mathrm{p}}$ is the screened ion plasma frequency, $\varepsilon_{\infty}$ is the high frequency relative permittivity $(\sim 10.88), \varepsilon_{\mathrm{m}}$ is the relative permittivity of the surrounding medium, $I_{\mathrm{n}}$ and $K_{\mathrm{n}}$ are the modified $n^{\text {th }}$ Bessel functions, $r_{\mathrm{NW}}$ is the NW radius, and $q$ is the wavevector which was calculated using the laser wavelength as $q=4 \pi / \lambda=0.023621 \mathrm{~nm}^{-1}$. The experimental values for the frequency shift between $\omega_{\mathrm{LO}}$ and $\omega_{\mathrm{SO}}$ are represented in Figure 6 as a function of the NW average diameter obtained from SEM analysis for several samples. Calculated $\omega_{\text {So }}$ values-using the set of Eqs. (4) for different environments such as air $\left(\varepsilon_{\mathrm{m}}=1\right)$ or oxide based compounds $\left(\mathrm{GaO}_{\mathrm{x}}, \mathrm{AsO}_{\mathrm{x}}\right.$, ...) whose relative permittivity was assumed to be around

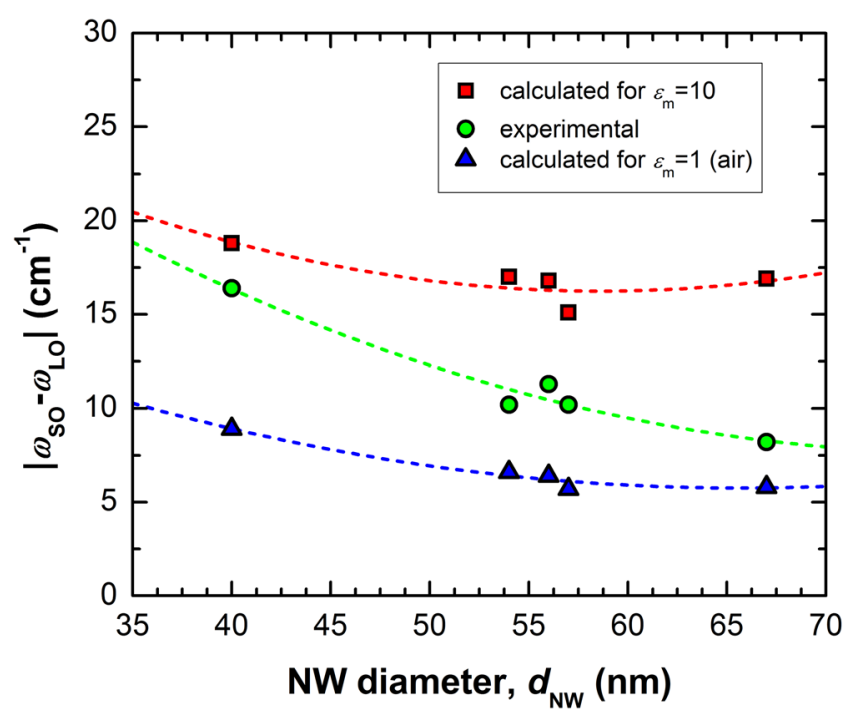

FIG. 6. SO to LO phonon frequency shift as a function of the NW diameter and the surrounding environment. Dotted lines are drawn to guide the eye.

$\varepsilon_{\mathrm{m}}=10$ - are also plotted in the same figure. As it can be observed in Figure 6, experimental data values are located between calculated values for air and oxide, meaning that the NWs were probably partially oxidized and capped with an oxide thin layer. Corresponding $\varepsilon_{\mathrm{m}}$ for each NW diameter can be roughly estimated using Eqs. (4) to calculate the effective $\varepsilon_{\mathrm{m}}$ value to fit experimental $\omega_{\mathrm{SO}}-\omega_{\mathrm{LO}}$ values, following the same procedure than used in Figure 6 for $\varepsilon_{\mathrm{m}}=1$ and $\varepsilon_{\mathrm{m}}=10$, and the obtained results are recorded in Table II.

Figure 6 shows as NW diameter increases (larger correlation lengths) the obtained frequency shift between SO and LO phonons decreases, which indicates NWs with larger diameters tend to be less oxidized along their surfaces due to their lower surface-to-volume ratio. Alternatively, different oxide coverage degree for different samples, possibly due to different post-growth storage conditions and ambient exposure times, is not discarded.

The SO mode detection in Raman spectroscopy is enhanced using backscattering configuration, because the surface potential perturbation along the NW axis is excited by a high component of the incident wavevector. The values of the wavevector responsible for the observation of the SO mode can be estimated by comparing experimental values of $\omega_{\mathrm{SO}}{ }^{\exp }$ to those calculated using Eqs. (4) for NWs with

TABLE II. Measured averaged NW diameters $\left(d_{\mathrm{NW}}\right)$, lengths $\left(l_{\mathrm{NW}}\right)$, and SO Raman shift $\left(\omega_{\mathrm{SO}}{ }^{\text {exp }}\right)$ for several samples; effective relative permittivity $\left(\varepsilon_{\mathrm{m}}\right), q r_{\mathrm{NW}}$ value, and length scale $(\lambda)$ calculated as a function of $d_{\mathrm{NW}}$.

\begin{tabular}{cccccc}
\hline \hline $\begin{array}{c}d_{\mathrm{NW}}=2 r_{\mathrm{NW}} \\
(\mathrm{nm})\end{array}$ & $\begin{array}{c}l_{\mathrm{NW}} \\
(\mu \mathrm{m})\end{array}$ & $\begin{array}{c}\omega_{\mathrm{SO}} \text { exp } \\
\left(\mathrm{cm}^{-1}\right)\end{array}$ & $\varepsilon_{\mathrm{m}}$ & $q r_{\mathrm{NW}}$ & $\begin{array}{c}\lambda \\
(\mu \mathrm{m})\end{array}$ \\
\hline 40 & 1.3 & 273.2 & 4.60 & 0.48 & 0.52 \\
54 & 0.4 & 279.4 & 2.07 & 0.64 & 0.72 \\
56 & 1.2 & 277.3 & 2.69 & 0.66 & 0.75 \\
57 & 0.4 & 277.3 & 2.78 & 0.67 & 0.76 \\
67 & 0.3 & 281.4 & 1.68 & 0.79 & 0.53 \\
\hline \hline
\end{tabular}




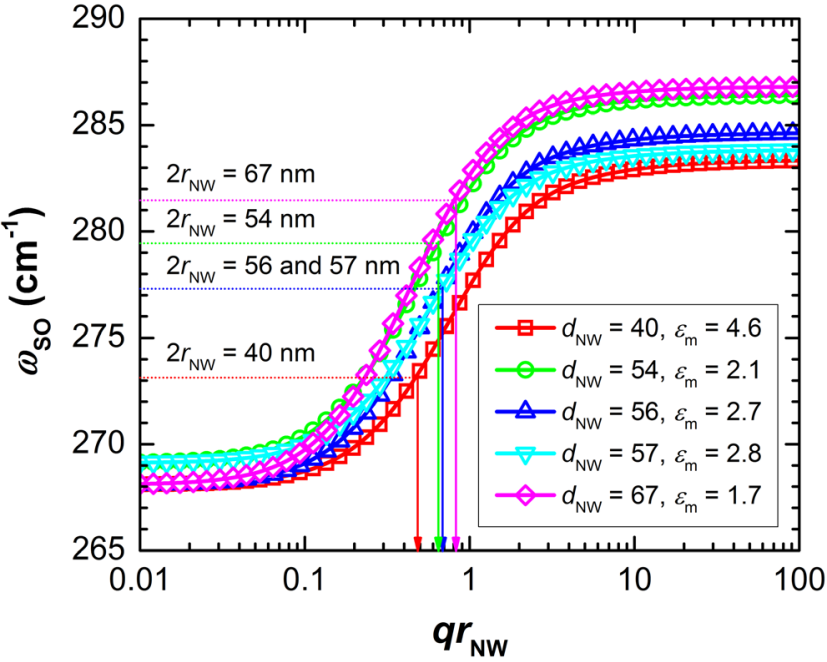

FIG. 7. Calculated SO Raman shift for single cylindrical GaAs NWs with different diameters and corresponding $\varepsilon_{\mathrm{m}}$ values. For a given radius $\left(r_{\mathrm{NW}}\right)$, $q^{\exp } r_{\mathrm{NW}}$ values are deduced from measured $\omega_{\mathrm{SO}}$ exp, following the model described in the text.

different radii in air ambient. As shown in Figure 7, corresponding $q^{\exp } r_{\mathrm{NW}}$ values are extracted from the intersection of $\omega_{\text {SO }}{ }^{\exp }$ (y axis) and calculated $\omega_{\text {SO }}$ values (plotted curve) for every NW dimensions and effective relative permittivity of the surrounding media, as pointed out by arrows in this figure. The obtained values for $q^{\exp } r_{\mathrm{NW}}$-shown in Table II-are located in a very short interval, in the range of 0.5 to 0.8 .

Many factors can induce the activation of the SO mode, the main ones being the surface roughness or changes in the NW diameter along the NW stem. Both are typically found in VLS growths due to changes in the catalyst size during growth. Distance between these kinds of surface defects can be roughly estimated using the length scale, usually defined
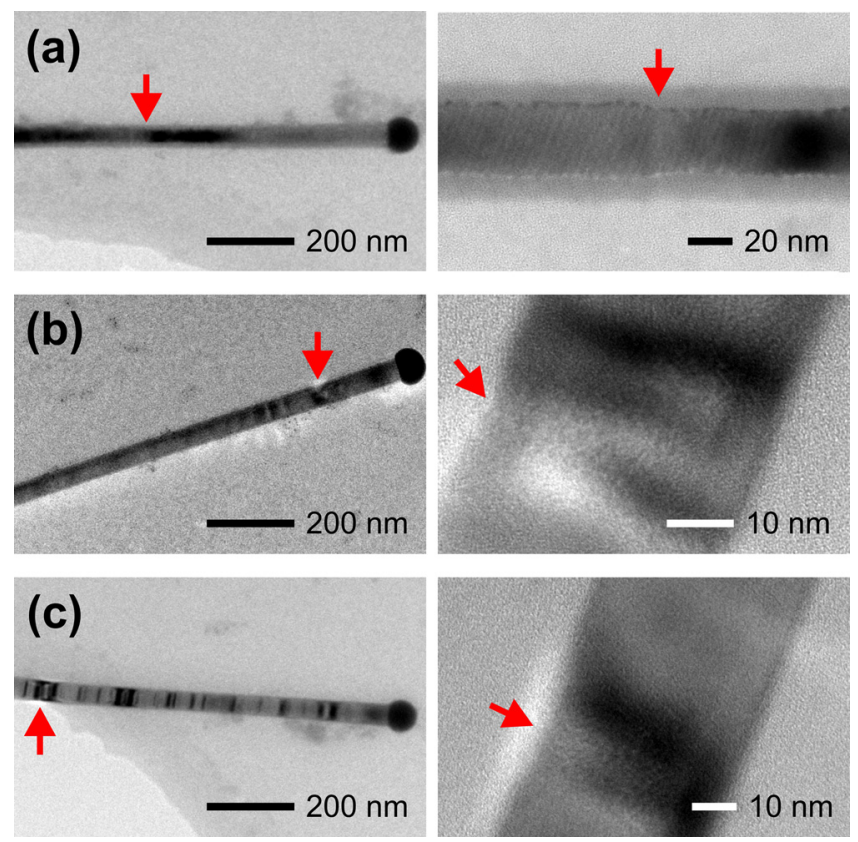

FIG. 8. TEM images showing (a) one surface step, (b) and (c) waist-style morphological defects found on some NWs. as $\lambda=2 \pi d_{\mathrm{NW}} / q^{\text {exp }} r_{\mathrm{NW}}{ }^{31}$ Calculated $\lambda$ values are included within a narrow interval, in the range 0.5 to $0.8 \mu \mathrm{m}$, as also shown in Table II.

The $\lambda$ value could be interpreted as the distance between steps along the NW surface, as previously observed in AIN $\mathrm{NWs}^{28}$ where symmetrical breaking responsible for the SO generation was mainly caused by the surface roughness modulation.

Figure 8 shows TEM overview images and their magnification, corresponding to three different NWs of about $40 \mathrm{~nm}$ diameter and $1.3 \mu \mathrm{m}$ length. These pictures highlight both the smooth surface of the NWs and the reduced number of morphological surface defects. As marked with arrows, the existence of one step (Figure 8(a)) and waist-style defects (Figures 8(b) and 8(c)) along the whole NW facets were hardly detected in some NWs.

Therefore, TEM confirms the low density of surface defects in GaAs NWs which is in good agreement with SO Raman results, where $\lambda$ takes values which are close to those measured by SEM for NW length $\left(l_{\mathrm{NW}}\right)$.

On the other hand, SO line width was analyzed as a function of the NW diameter. There was no significant difference between the full width at half maximum values obtained for NWs with $40 \mathrm{~nm}$ of diameter $\left(11.0 \mathrm{~cm}^{-1}\right)$ and those obtained for NW with $67 \mathrm{~nm}\left(17 \mathrm{~cm}^{-1}\right)$, thus meaning SO phonons had similar decay channels and their lifetime was approximately in the same range. ${ }^{31}$

\section{CONCLUSIONS}

Morphological and structural properties of GaAs NWs grown by Ga-assisted CBE were studied by SEM, microRaman spectroscopy, and TEM. Growth conditions used in this work produced NWs with an exceptional crystalline quality corresponding to a pure $\mathrm{ZB}$ phase.

Raman spectra obtained in backscattering configuration evidenced the presence of a SO phonon mode located in between the TO and LO modes, and whose frequency depends either on the NW diameter or on the oxide coverage. LO shape analysis allowed to obtain the value for the confinement coefficient in GaAs NWs, taking values around $\alpha=23 \mathrm{~nm}^{-1}$, characteristic for this material.

The frequencies of SO mode shifted downwards as NW diameter decreased and/or NW oxide thickness increased. Wavevector responsible for the SO activation in NWs was calculated in the case of air ambient, showing length scales between 0.5 and $0.8 \mu \mathrm{m}$ - which are similar to the NW length - and indicating the high uniformity of the cross section size during the NW growth.

\section{ACKNOWLEDGMENTS}

G. Shen and C. García Núñez acknowledge the support of the Alabama EPSCoR GRSP and the FPI-MINECO 2012 grant, respectively. This work used the Central Analytical Facility and SIdI, which are supported by the University of Alabama and Universidad Autónoma de Madrid, respectively. This research was supported by the Spanish Ministry of Economy and Competitiveness TEC2010-20796 project. 
${ }^{1}$ M. T. Björk, B. J. Ohlsson, C. Thelander, A. I. Persson, K. Deppert, L. R. Wallenberg, and L. Samuelson, Appl. Phys. Lett. 81, 4458 (2002).

${ }^{2}$ C. Thelander, T. Mårtensson, M. T. Björk, B. J. Ohlsson, M. W. Larsson, L. R. Wallenberg, and L. Samuelson, Appl. Phys. Lett. 83, 2052 (2003).

${ }^{3}$ H. Pettersson, J. Trägårdh, A. I. Persson, L. Landin, D. Hessman, and L. Samuelson, Nano Lett. 6, 229 (2006).

${ }^{4}$ L. Samuelson, Mater. Today 6, 22 (2003).

${ }^{5}$ G. E. Cirlin, V. G. Dubrovskii, Y. B. Samsonenko, A. D. Bouravleuv, K. Durose, Y. Y. Proskuryakov, B. Mendes, L. Bowen, M. A. Kaliteevski, R. A. Abram, and D. Zeze, Phys. Rev. B 82, 035302 (2010).

${ }^{6} \mathrm{H}$. Huang, X. Ren, X. Ye, J. Guo, Q. Wang, Y. Yang, S. Cai, and Y. Huang, Nano Lett. 10, 64 (2010).

${ }^{7}$ A. I. Persson, M. W. Larsson, S. Stenström, B. J. Ohlsson, L. Samuelson, and L. R. Wallenberg, Nature Mater. 3, 677 (2004).

${ }^{8}$ R. S. Wagner and W. C. Ellis, Appl. Phys. Lett. 4, 89 (1964).

${ }^{9}$ P. Yang, R. Yan, and M. Fardy, Nano Lett. 10, 1529 (2010).

${ }^{10}$ W. Lu and C. M. Lieber, Nature Mater. 6, 841 (2007).

${ }^{11}$ L. E. Fröberg, W. Seifert, and J. Johansson, Phys. Rev. B 76, 153401 (2007).

${ }^{12}$ J. B. Hannon, S. Kodambaka, F. M. Ross, and R. M. Tromp, Nature 440, 69 (2006).

${ }^{13}$ D. E. Perea, J. E. Allen, S. J. May, B. W. Wessels, D. N. Seidman, and L. J. Lauhon, Nano Lett. 6, 181 (2006).

${ }^{14}$ Y. H. Kim, D. W. Park, and S. J. Lee, Appl. Phys. Lett. 100, 033117 (2012).

${ }^{15}$ S. Plissard, K. A. Dick, G. Larrieu, S. Godey, A. Addad, X. Wallart, and P. Caroff, Nanotechnology 21, 385602 (2010).

${ }^{16}$ C. García Núñez, A. F. Braña, J. L. Pau, D. Ghita, B. J. García, G. Shen, D. S. Wilbert, S. M. Kim, and P. Kung, J. Cryst. Growth 372, 205 (2013).

${ }^{17}$ G. E. Cirlin, V. G. Dubrovskii, I. P. Soshnikov, N. V. Sibirev, Y. B. Samsonenko, A. D. Bouravleuv, J. C. Harmand, and F. Glas, Phys. Status Solidi (RRL) 3, 112 (2009).
${ }^{18}$ D. Spirkoska, G. Abstreiter, and A. Fontcuberta i Morral, Nanotechnology 19, 435704 (2008).

${ }^{19}$ S. Piscanec, M. Cantoro, A. C. Ferrari, J. A. Zapien, Y. Lifshitz, S. T. Lee, S. Hofmann, and J. Robertson, Phys. Rev. B 68, 241312 (2003).

${ }^{20}$ K. W. Adu, H. R. Gutiérrez, U. J. Kim, G. U. Sumanasekera, and P. C. Eklund, Nano Lett. 5, 409 (2005).

${ }^{21}$ K. W. Adu, Q. Xiong, H. R. Gutierrez, G. Chen, and P. C. Eklund, Appl. Phys. A 85, 287 (2006).

${ }^{22}$ K. K. Tiong, P. M. Amirtharaj, F. H. Pollak, and D. E. Aspnes, Appl. Phys. Lett. 44, 122 (1984).

${ }^{23}$ D. Spirkoska, J. Arbiol, A. Gustafsson, S. Conesa-Boj, F. Glas, I. Zardo, M. Heigoldt, M. H. Gass, A. L. Bleloch, S. Estrade, M. Kaniber, J. Rossler, F. Peiro, J. R. Morante, G. Abstreiter, L. Samuelson, and A. Fontcuberta i Morral, Phys. Rev. B 80, 245325 (2009).

${ }^{24}$ I. H. Campbell and P. M. Fauchet, Solid State Commun. 58, 739 (1986).

${ }^{25}$ N. Begum, M. Piccin, F. Jabeen, G. Bais, S. Rubini, F. Martelli, and A. S. Bhatti, J. Appl. Phys. 104, 104311 (2008).

${ }^{26}$ R. Fuchs and K. L. Kliewer, Phys. Rev. 140, A2076 (1965).

${ }^{27}$ S. E. Wu, S. Dhara, T. H. Hsueh, Y. F. Lai, C. Y. Wang, and C. P. Liu, J. Raman Spectrosc. 40, 2044 (2009).

${ }^{28}$ S. Sahoo, S. Dhara, A. K. Arora, R. Krishnan, P. Chandramohan, and M. P. Srinivasan, Appl. Phys. Lett. 96, 103113 (2010).

${ }^{29}$ P. Sahoo, S. Dhara, S. Dash, A. K. Tyagi, and B. Raj, Int. J. Nanotechnol. 7, 823 (2010).

${ }^{30}$ S. Sahoo, M. S. Hu, C. W. Hsu, C. T. Wu, K. H. Chen, L. C. Chen, A. K. Arora, and S. Dhara, Appl. Phys. Lett. 93, 233116 (2008).

${ }^{31}$ R. Gupta, Q. Xiong, G. D. Mahan, and P. C. Eklund, Nano Lett. 3, 1745 (2003).

${ }^{32}$ H. Richter, Z. P. Wang, and L. Ley, Solid State Commun. 39, 625 (1981).

${ }^{33}$ C. Kittel, Introduction to Solid State Physics, 7th ed. (Wiley, New York, 2003), Chap. 4.

${ }^{34}$ K. W. Adu, H. R. Gutierrez, U. J. Kim, and P. C. Eklund, Phys. Rev. B 73, 155333 (2006). 